\title{
Primer reporte de bacterias y dinoflagelados marinos luminiscentes del Parque Nacional Isla del Coco, Costa Rica
}

\author{
Rebeca Rojas-Alfaro ${ }^{1 *}$, Rodolfo Umaña-Castro ${ }^{2}$, Norman Rojas-Campos ${ }^{3}$ \\ \& Maribel Vargas-Montero ${ }^{1,4}$ \\ 1. Centro de Investigación en Estructuras Microscópicas, Universidad de Costa Rica, San José, Costa Rica; \\ mariarebeca.rojas@ucr.ac.cr, rebeca.rojas.alfaro@gmail.com
}

2. Laboratorio de Análisis Genómico, Escuela de Ciencias Biológicas, Universidad Nacional, Heredia, Costa Rica; rodolfo.umana.castro@una.cr

3. Centro de Investigación en Enfermedades Tropicales, Facultad de Microbiología, Universidad de Costa Rica, San José, Costa Rica; norman.rojas@ucr.ac.cr

4. Facultad de Microbiología, Universidad de Costa Rica; maribelle.vargas@ucr.ac.cr * Correspondence

Recibido 05-II-2019. Corregido 25-IV-2019. Aceptado 05-VII-2019.

\begin{abstract}
First report of marine luminescent bacteria and dinoflagellates from Isla del Coco National Park, Costa Rica. Introduction: Bioluminescence is the ability of certain organisms to transform chemical energy into light energy through various biochemical processes. Objective: Isolation and identification for the first time of luminescent bacteria of superficial marine water, and the identification of marine luminescent dinoflagellates of Isla del Coco National Park, Costa Rica. Methods: Samples of seawater obtained by diving at $20 \mathrm{~m}$ and at a surface level of 13 sites were collected. On the other hand, phytoplankton samples collected from the surface up to $30 \mathrm{~m}$ deep were analyzed in the surroundings of 8 sites of Cocos Island, and several luminescent species belonging to the genera Ornithocercus and Ceratocorys were determined. Results: Seven luminescent bacterial isolates were obtained, they were identified and characterized biochemically by means of an automated platform (Vitek) with high levels of confidence, they were taxonomically located within the genus Vibrio, 2 species: $V$. alginolyticus and $V$. parahaemolyticus, in addition, some isolates presented resistance to the antibiotic ampicillin and $100 \%$ hemolytic capacity. This research shows evidence of the presence of marine microscopic species in Cocos Island, Costa Rica, capable of presenting the phenomenon of luminescence, so that further study would be relevant in terms of the importance of these microorganisms in the production of metabolites secondary and as indicators of harmful algal blooms, so it is necessary to conduct more scientific research to determine their biotechnological potential. Conclusions: In the same way, the results obtained in this investigation suggest expanding the collection and isolation of luminescent microorganisms, accompanied by a biochemical and molecular characterization, in order to explore the microbial diversity associated with luminescence events and determine the environments in which that these species develop.
\end{abstract}

Key words: Luminescence, marine bacteria, dinoflagellates, Gram-negative bacilli, Cocos Island.

Rojas-Alfaro, R., Umaña-Castro, R., Rojas-Campos, N., \& Vargas-Montero, M. (2020). Primer reporte de bacterias y dinoflagelados marinos luminiscentes del Parque Nacional Isla del Coco, Costa Rica. Revista de Biología Tropical, 68(Supl. 1), S213-S224.

Los océanos representan alrededor del $71 \%$ de la superficie del planeta y los estudios han demostrado que el ambiente marino contiene gran cantidad de especies bacterianas que recientemente se están caracterizando (Kola
\& Masilamani, 2017). Costa Rica se ubica en una de las zonas de alta diversidad de especies a nivel mundial, por ser un corredor biológico entre el norte y sur del continente americano. Posee una superficie marina de $568054 \mathrm{~km}^{2}$, 
de las cuales se han identificado cerca de 7000 especies marinas, que representan un $6.5 \%$ de las especies registradas a nivel internacional, es uno de los países con más diversidad por $\mathrm{Km}^{2}$ (Wehrtmann et al., 2009; Cortés, 2017). La costa Pacífica posee más del doble de especies marinas que las reportadas en el Caribe; sin embargo, esta estimación no comprende a la comunidad de microorganismos (Wehrtmann et al., 2009; Alvarado, Herrera, Corrales, Asch, \& Paaby, 2011; Cortés, 2012, 2016 a, b). La mayoría de las investigaciones de biodiversidad se han enfocado en grandes mamíferos marinos, vertebrados como peces y algunos invertebrados como corales entre otros (Wehrtmann \& Cortés, 2009). Sin embargo, a pesar de la alta diversidad de ecosistemas marinos, en el país existen vacíos en el tema de conservación a nivel de la comunidad microbiana y fitoplanctónica, estos deben ser abordados para asegurar la conservación del patrimonio natural y genético.

Dentro de las bacterias marinas existe un grupo bacterias luminiscentes, capaces de transformar la energía química en energía lumínica mediante un proceso bioquímico (Kahlke \& Umbers, 2016). De 700 géneros de organismos marinos que producen bioluminiscencia, el $80 \%$ corresponden a relaciones simbióticas con bacterias y su distribución geográfica abarca desde los polos hasta aguas tropicales. Las bacterias se han reportado como organismos de vida libre, asociados a peces y cefalópodos o formando relaciones simbióticas en órganos lumínicos de organismos como el calamar Euprymna scolopes (Kola \& Masilamani, 2017) y en peces como Photoblepharon sp. y Leiognathus sp., y en medusas como Atolla sp. (Witze, 2018). Los dinoflagelados son microalgas pertenecientes al grupo del fitoplancton, y junto a las diatomeas son uno de los grupos más importantes en el ecosistema debido a la producción de potentes toxinas. Además, estos organismos son los mayores productores de floraciones algales nocivas, siendo algunos de estos luminiscentes, incluso se hace referencia de un dinoflagelado bioluminiscente de aguas marinas tropicales (Noctiluca miliaris) (Villalobos-Solé, 1967).

En el Parque Nacional Isla del Coco (PNIC), durante una expedición científica, se analizaron muestras de fito y zooplancton, logrando reportar 75 especies de bacterias y más de 100 especies de microalgas pertenecientes al fitoplancton (Cortés-Núñez, Acuña-González, Lizano-Rodríguez, MoralesRamírez, \& Alfaro-Martínez, 2009). En Costa Rica se han realizado estudios metagenómicos dirigidos a bacterias marinas, reportándose en la columna de agua los géneros Prochlorococcus, Synechococcus, Pelagibacter, Shewanella, y Burkholderia cerca de la Isla del Coco, Roca Sucia, a una profundidad de $30 \mathrm{~m}$ (Rusch et al., 2007). La primera referencia sobre bacterias luminiscentes aisladas del ecosistema marino costarricense fue presentada en el VII Congreso Nacional de Biología por VegaCorrales, Marín-Vindas y Rojas-Alfaro (2014), quienes realizaron el primer reporte nacional sobre el aislamiento de bacterias luminiscentes del Golfo de Nicoya. Destacan otros reportes como los de Marín-Vindas Vega-Corrales, Umaña-Castro y Rojas-Alfaro (2015, 2017), Marín-Vindas, Vega-Corrales y Rojas-Alfaro (2015) y Rojas-Alfaro (2016) indicando la presencia de bacterias marinas luminiscentes de los géneros Vibrio y Photobacterium en la costa del Pacífico costarricense. En Costa Rica desde 1995 se han realizado estudios sobre dinoflagelados dirigidos hacia la identificación taxonómica y en la amenaza que representan debido a la producción de las floraciones algales en las áreas marinas (Víquez \& Hargraves, 1995; Vargas-Montero \& Freer, 2003; VargasMontero, Freer, Guzmán, \& Vargas, 2008). Actualmente, no hay referencias sobre dinoflagelados luminiscentes encontrados en el medio marino costarricense.

Por tanto, esta investigación tuvo como objetivo aislar e identificar por primera vez bacterias y dinoflagelados luminiscentes de agua marina superficial entre los 3 a $30 \mathrm{~m}$ de profundidad del Parque Nacional Isla del Coco, Costa Rica. 


\section{MATERIALES Y MÉTODOS}

Área de estudio: Para bacterias bioluminiscentes en junio del 2017 se recolectaron muestras de agua marina superficial y mediante buceo de hasta $30 \mathrm{~m}$ de 13 zonas del PNIC (Fig. 1). Para las colectas de agua se utilizaron botellas ámbar estériles de $100 \mathrm{~mL}$. Seguidamente, las muestras de agua se almacenaron y transportaron en hielo en un lapso menor a 4 h hasta la Estación Bahía Wafer, PNIC, Costa Rica. Las muestras de fitoplancton se recolectaron en 8 zonas del PNIC, desde la superficie hasta los 30 metros de profundidad utilizando botellas Niskin de $1 \mathrm{~L}$ de capacidad, así como realizando buceos sobre parches de coral utilizando una red de 20 um de los de malla, fijándose inmediatamente con glutraldehído al $2 \%$ en la estación de Bahía Wafer (Vargas-Montero et al., 2008), luego fueron transportadas al Laboratorio de Fitoplancton Marino y Costero del Centro de Investigación en Estructuras Microscópicas (CIEMic), de la Universidad de Costa Rica (Tabla 1).

Procesamiento de la muestra y análisis microbiológico: Las muestras de agua marina colectadas fueron procesadas y analizadas en un cuarto adaptado para ensayos microbiológicos en la Estación Bahía Wafer, PNIC. La metodología utilizada fue la propuesta por Bagordo et al. (2012), con las siguientes modificaciones: se tomaron alícuotas de $100 \mathrm{~mL}$ de cada muestra y se filtraron a través de filtros de nitrato de celulosa de $0.45 \mu \mathrm{m}$ de tamaño de poro y de $47 \mathrm{~mm}$ de diámetro (MiliPore ${ }^{\circledR}$ ).

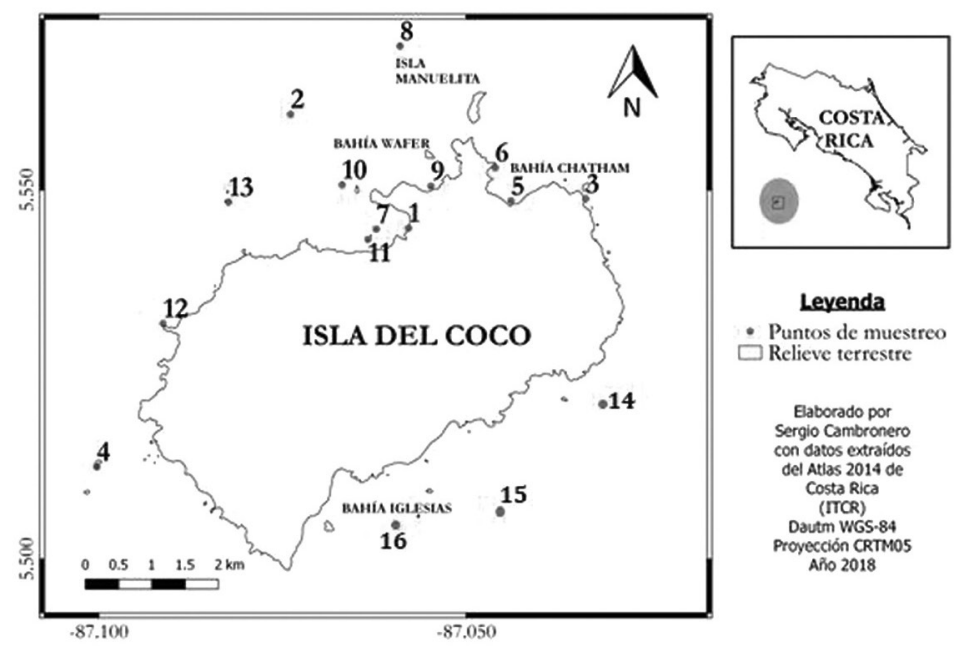

Fig. 1. Mapa de la distribución de las zonas de muestreo de aislados bacterianos luminiscentes de la Isla del Coco, Costa Rica. Muestras superficiales: (1) Bahía Wafer, (2) Roca Vikinga, (3) Punta Ulloa, (5) Bahía Chatam, (6) Fondeo del barco Sea Hunter, (7) fondeo del barco Okeanos Aggressor II, (9) Bahía Weston, (10) Punta Presidio, (11) Roca Gissler. Muestras obtenidas por buceo: (4) Roca 2 amigos (20 m), (8) Manuelita profundo (20 m), (12) Punta María (20 m) y (13) Roca Sucia $(30 \mathrm{~m})$. Zonas de muestreo de dinoflagelados marinos luminiscentes, muestras superficiales: (4) Roca 2 amigos, (11) Roca Gissler y (12) Punta María. Muestras obtenidas por buceo: (1) Coral de Bahía Wafer (3 m), (5) Coral de Bahía Chatam (5 m), (14) Cabo Atrevido (5 m), (15) Bajo Alcyone (30 m) and (16) Bahía Iglesias (20 m). Entre paréntesis la profundidad en metros en que se recolectó la muestra.

Fig. 1. Map of the distribution of the sampling zones of luminescent bacteria from Cocos Island, Costa Rica. Surface samples: (1) Wafer Bay, (2) Viking Rock, (3) Ulloa Point, (5) Chatam Bay, (6) Anchorage of the Sea Hunter ship, (7) Anchorage of the Okeanos Agresor ship, (9) Weston Bay, (10) Presidio Point, (11) Gissler Rock. Samples obtained by diving: (4) 2 friends Rock (20 m), (8) Manuelita deep (20 m), (12) María Point (20 m) and (13) Dirty Rock (30 m).

Sampling zones of marine bioluminescent dinoflagenates, Surface samples: (4) Two friends Rock, (11) Gissler Rock and (12) María Point. Diving samples: (1) Coral of Wafer Bay (3 m), (5) Coral of Chatam Bay (5 m), (14) Atrevido Cape (5 m), (15) Bajo Alcyone (30m), (16) Churches Bay (20m). In parentheses the depth in meters in which the sample was collected. 
TABLA 1

Sitos de muestreo de aislados bacterianos y colecta de fitoplancton en la Isla del Coco, Costa Rica

TABLE 1

Sampling sites for bacterial isolates and phytoplanckton samples from Isla del Coco, Costa Rica

\begin{tabular}{|c|c|c|}
\hline \multirow{2}{*}{ Sitios de muestreo } & \multicolumn{2}{|c|}{ Ubicación GPS } \\
\hline & $\mathrm{N}$ & W \\
\hline \multicolumn{3}{|c|}{ Muestras a nivel Superficial (1 m de profundidad) } \\
\hline Bahía Wafer & $05^{\circ} 33^{\prime} 12^{\prime \prime}$ & $87^{\prime} 02^{\prime} 28^{\prime \prime}$ \\
\hline Roca Vikinga & $05^{\circ} 32^{\prime} 09^{\prime \prime}$ & $87^{\circ} 03$ ' $86^{\prime \prime}$ \\
\hline Punta Ulloa & $05^{\circ} 33^{\prime} 10^{\prime \prime}$ & $87^{\circ} 02^{\prime} 21^{\prime \prime}$ \\
\hline Bahía Chatham & $05^{\circ} 33^{\prime} 01^{\prime \prime}$ & $87^{\prime} 02^{\prime} 30^{\prime \prime}$ \\
\hline Bahía Weston & $05^{\circ} 33^{\prime} 03^{\prime \prime}$ & $87^{\circ} 03 ’ 02^{\prime \prime}$ \\
\hline Punta Presidio & $05^{\circ} 33^{\prime} 12^{\prime \prime}$ & $82^{\prime} 02^{\prime} 55^{\prime \prime}$ \\
\hline Roca Gissler & $05^{\circ} 32^{\prime} 06^{\prime \prime}$ & $87^{\circ} 03^{\prime} 07^{\prime \prime}$ \\
\hline Fondeo Sea Hunter & $05^{\circ} 33^{\prime} 09^{\prime \prime}$ & $87^{\prime} 02^{\prime} 24^{\prime \prime}$ \\
\hline Fondeo Okeanos Aggressor II & $05^{\circ} 33^{\prime} 08^{\prime \prime}$ & $87^{\prime} 02^{\prime} 36^{\prime \prime}$ \\
\hline \multicolumn{3}{|l|}{ Muestras obtenidas por buceo } \\
\hline Manuelita profundo (20 m) & $05^{\circ} 33^{\prime} 07^{\prime \prime}$ & $87^{\circ} 02^{\prime} 09^{\prime \prime}$ \\
\hline Roca 2 amigos grande $(20 \mathrm{~m})$ & $05^{\circ} 30^{\prime} 07^{\prime \prime}$ & $87^{\circ} 05^{\prime} 10^{\prime \prime}$ \\
\hline Punta María (20 m) & $05^{\circ} 31^{\prime} 09^{\prime \prime}$ & $87^{\circ} 05^{\prime} 06^{\prime \prime}$ \\
\hline Roca Sucia (30 m) & $05^{\circ} 32^{\prime} 09^{\prime \prime}$ & $87^{\circ} 04^{\prime} 09^{\prime \prime}$ \\
\hline Cabo atrevido $(5 \mathrm{~m})$ & $05^{\circ} 31^{\prime} 99^{\prime \prime}$ & $87^{\circ} 01^{\prime} 55^{\prime \prime}$ \\
\hline Bajo Alcyone (30 m) & $05^{\circ} 30^{\prime} 99^{\prime \prime}$ & $87^{\circ} 01^{\prime} 55^{\prime \prime}$ \\
\hline Bahía Iglesias (30 m) & $05^{\circ} 30^{\prime} 64^{\prime \prime}$ & $87^{\circ} 03^{\prime} 92^{\prime \prime}$ \\
\hline Coral de Bahía Wafer (3 m) & $05^{\circ} 33^{\prime} 12^{\prime \prime}$ & $87^{\prime} 02^{\prime} 28^{\prime \prime}$ \\
\hline Coral de Bahía Chatham (5 m) & $05^{\circ} 33^{\prime} 01^{\prime \prime}$ & 87’02’30” \\
\hline
\end{tabular}

* Entre paréntesis la profundidad en metros en que se recolectó la muestra.

Seguidamente, los filtros se colocaron en placas Petri con agar Zobell (Khambholja \& Kalia, 2016) y se incubaron en oscuridad a temperatura ambiente local aproximada de 26-27 ${ }^{\circ} \mathrm{C}$ durante $24 \mathrm{~h}$. Posterior al período de incubación, se seleccionaron las colonias con una luminiscencia basal de $100 \%$, de forma visual en la oscuridad.

Adicionalmente, se realizaron tinciones de Gram (Bagordo, et al., 2012) y posteriormente los aislados fueron identificados. Se realizó el antibiograma mediante el sistema automatizado Vitek 2 Compact (bioMerieux, Francia). Y se determinó la actividad hemolítica mediante la inoculación por estría a cultivos frescos $(18$ a $24 \mathrm{~h})$ de agar sangre $(10 \%)$ de cada aislamiento luminiscente. Las placas fueron incubadas a $30{ }^{\circ} \mathrm{C}$ durante $24 \mathrm{~h}$ y fueron almacenadas en caldo BHI (Caldo infusión cerebro corazón) $\left(\mathrm{OXOID}^{\circledR}\right.$ ) con $20 \%$ glicerol a $-80{ }^{\circ} \mathrm{C}$ (Chunga, 2018) en el Laboratorio de Bacteriología Médica de la Facultad de Microbiología, Universidad de Costa Rica, San José, Costa Rica. La actividad hemolítica se determinó por la presencia de halos (zonas claras) según tipo alfa, beta o gamma hemólisis evidenciada alrededor de la estría (Martins, 2010).

Procesamiento de la muestra de fitoplancton: Las muestras de fitoplancton se sedimentaron y se procesaron en el laboratorio para ser analizadas en un microscopio de luz invertida, marca Olympus ${ }^{\circledR}$ equipado con una cámara DP-70. Las muestras más representativas se procesaron para ser fotografiadas utilizando un microscopio electrónico de barrido marca 
Hitachi, modelo S-570. El procesamiento realizado consistió en extraer aproximadamente $1 \mathrm{~mL}$ de la muestra sedimentada, la cual fue procesada siguiendo la metodología propuesta por Watson, Mckee \& Merrel (1980) para la digestión de membranas, utilizando un detergente no iónico (Tritón X-100 ${ }^{\circledR}$ ). La separación de la muestra se llevó a cabo a través de gradientes de sucrosa, lo que permitió aislar los dinoflagelados de los residuos presentes en la muestra. Las muestras procesadas se fijaron en una membrana de Poli-L-Lisina de acuerdo a Mazia, Schatten \& Sale (1975) y fueron cubiertas con una fina capa de oro-paladio mediante el cobertor iónico Geiko IB3, para facilitar su visualización en el microscopio de barrido del CIEMic. Una vez fotografiadas se procedió a identificar las especies con la literatura más actualizada.

\section{RESULTADOS}

Se obtuvieron siete aislados de bacterias luminiscentes de agua marina: seis a nivel superficial y una mediante buceo de roca Dos Amigos (20 m de profundidad) de la Isla del Coco, Costa Rica (Fig. 2, Tabla 1, Tabla 2). En cuanto a la morfología macroscópica

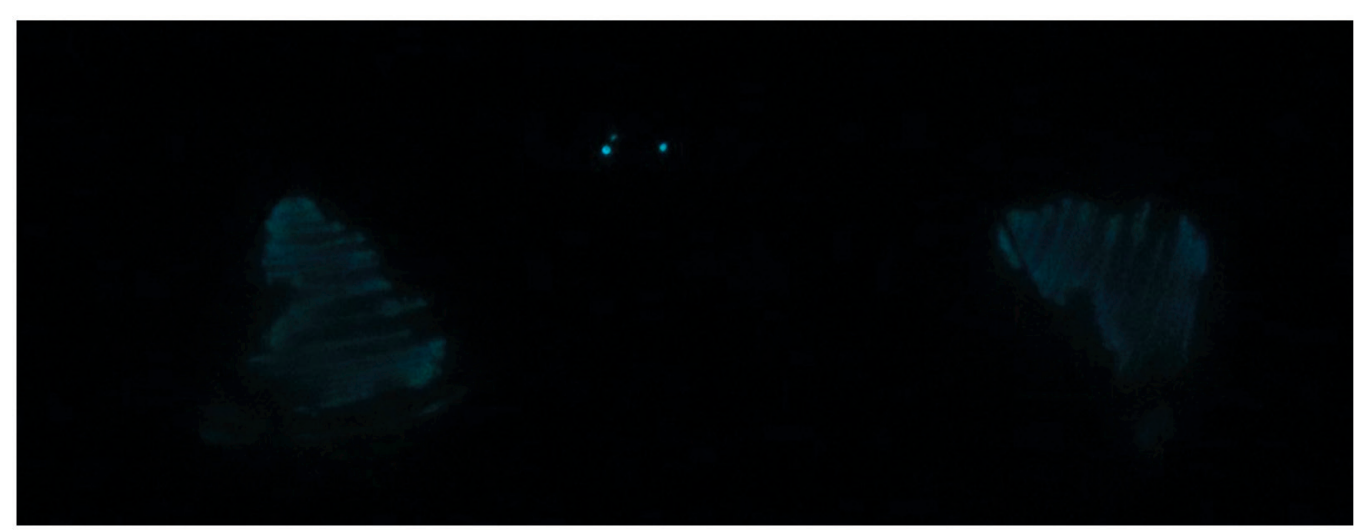

Fig. 2. Fotografía de bacterias luminiscentes cultivadas en agar Zobell aisladas de la Isla del Coco, Costa Rica, observadas en oscuridad total y sin exposición de ningún tipo de fuente de luz. Fotografía por: Guillermo Blanco Segura.

Fig. 2. Photograph of luminescent bacteria cultured on Zobell agar isolated from Cocos Island, Costa Rica, observed in total darkness and without exposure of any type of light source. Photography by: Guillermo Blanco Segura.

TABLA 2

Identificación bioquímica mediante Vitek 2 Compact (bioMerieux ${ }^{\circledR}$, Francia) de las bacterias luminiscentes aisladas de la Isla del Coco, Costa Rica

TABLE 2

Biochemical identification by Vitek 2 Compact (bioMerieux ${ }^{\circledR}$, France) of luminescent bacteria isolated from Cocos Island, Costa Rica

\begin{tabular}{cllc} 
Código cepa & \multicolumn{1}{c}{ Origen de la cepa } & \multicolumn{1}{c}{ Identificación Vitek 2 } & Nivel de confianza (\%) \\
CL 1 & Sitio 1: Bahía Wafer & Vibrio alginolyticus & 98 \\
CL 2 & Sitio 2: Roca Vikinga & Vibrio parahaemolyticus & 96 \\
CL 3 & Sitio 3: Punta Ulloa & Vibrio alginolyticus & 99 \\
CL 4 & Sitio 4: Roca 2 Amigos & Vibrio parahaemolyticus & 97 \\
CL 5 & Sitio 1: Bahía Wafer & Vibrio alginolyticus & 97 \\
CL 6 & Sitio 6: Fondeo Sea Hunter & Vibrio parahaemolyticus & 99 \\
CL 7 & Sitio 7:Fondeo Okeanos Aggressor II & Vibrio parahaemolyticus & 99 \\
\hline
\end{tabular}


se observaron colonias blancas translúcidas pequeñas $(<10 \mathrm{~mm})$ redondas con borde liso y microscópicamente todas se evidenciaron como bacilo Gram negativo (BGN).

El análisis bioquímico mediante Vitek 2 Compact (bioMerieux ${ }^{\circledR}$, Francia) logró ubicar taxonómicamente las aislados bacterianos luminiscentes de la Isla del Coco, Costa Rica. Estas se agruparon en el género Vibrio, se identificaron dos especies: $V$. alginolyticus y $V$. parahaemolyticus. Estos resultados están respaldados por un nivel de confianza ( $>96 \%$ ) (Tabla 2). Los aislados fueron $100 \%$ sensibles a los antibióticos ensayados, con excepción de la ampicilina, donde la sensibilidad fue de $57 \%$ (4/7 aislados) (Tabla 3).

En cuanto a la actividad hemolítica la totalidad de los aislados bacterianos luminiscentes fueron positivos, se evidenciaron halos de hemólisis de 7 hasta $10 \mathrm{~mm}$, en su mayoría los halos que rodearon a las colonias fueron totalmente transparentes, debido a la destrucción total de la membrana del eritrocito, característico de hemólisis completa (tipo beta), a diferencia de CL 3 que mostró una hemólisis parcial (tipo alfa) es decir la zona de crecimiento apareció rodeado de un halo de color verdoso producto de la lisis parcial del glóbulo rojo (Tabla 4).

En la Isla del Coco se encontraron varias familias de dinoflagelados productoras de bioluminiscencia (Fig. 3), observándose diferentes especies luminiscentes de los géneros Ornithocercus perteneciente al orden Dinophysiales, a la familia Dinophyceae y el género Ceratocorys perteneciente al orden Gonyaulacales, a la familia Ceratocoryaceae, esta última especie distinguida por no poseer cloroplastos, son heterotróficos, con la capacidad de mantener simbiontes fotosintéticos ubicados en su parte apical. Las especies encontradas fueron Ornithocercus steinii, Ornithocercus magnificus, Cerathocorys armata y Cerathocorys horrida.

TABLA 3

Antibiograma mediante Vitek 2 Compact (bioMerieux ${ }^{\circledR}$, Francia) de las bacterias luminiscentes aisladas de la Isla del Coco, Costa Rica. CMI: Concentración mínima inhibitoria parcial $(\mu \mathrm{g} / \mathrm{ml})$. Interpretación: S (susceptible) y R (resistente). BLEE: betalactamasa de espectro extendido

TABLE 3

Antibiogram using Vitek 2 Compact (bioMerieux ${ }^{\circledR}$, France) of luminescent bacteria isolated from Cocos Island Costa Rica. MIC: Minimum partial inhibitory concentration ( $\mu \mathrm{g} / \mathrm{ml})$. Interpretation: S (susceptible) and R (resistant). ESBL: extended-spectrum beta-lactamase

\begin{tabular}{lcccccccccccccc}
\multicolumn{1}{c}{ Antibiótico/Cepa } & \multicolumn{1}{c}{ CL 1 } & \multicolumn{2}{c}{ CL 2 } & \multicolumn{1}{c}{ CL 3 } & \multicolumn{2}{c}{ CL 4 } & \multicolumn{2}{c}{ CL 5 } & \multicolumn{2}{c}{ CL 6 } & CL 7 \\
Concentración e interpretación & CMI & S/R & CMI & S/R & CMI & S/R & CMI & S/R & CMI & S/R & CMI & S/R & CMI & S/R \\
BLEE & - & - & - & - & - & - & - & - & - & - & - & - & - & - \\
Ampicilina & $\leq 2$ & $\mathrm{~S}$ & $\leq 2$ & $\mathrm{~S}$ & $\geq 32$ & $\mathrm{R}$ & $\geq 32$ & $\mathrm{R}$ & $\geq 32$ & $\mathrm{R}$ & $\geq 32$ & $\mathrm{R}$ & $\leq 2$ & $\mathrm{~S}$ \\
Ampicilina/Sulbactam & $\leq 2$ & $\mathrm{~S}$ & $\leq 2$ & $\mathrm{~S}$ & $\leq 2$ & $\mathrm{~S}$ & $\leq 2$ & $\mathrm{~S}$ & $\leq 2$ & $\mathrm{~S}$ & $\leq 2$ & $\mathrm{~S}$ & $\leq 2$ & $\mathrm{~S}$ \\
Piperacilina/ Tazobactam & $\leq 4$ & $\mathrm{~S}$ & - & - & $\leq 4$ & $\mathrm{~S}$ & - & - & $\leq 4$ & $\mathrm{~S}$ & $\leq 2$ & $\mathrm{~S}$ & $\leq 2$ & $\mathrm{~S}$ \\
Ceftazidima & $\leq 1$ & $\mathrm{~S}$ & $\leq 1$ & $\mathrm{~S}$ & $\leq 1$ & $\mathrm{~S}$ & $\leq 1$ & $\mathrm{~S}$ & $\leq 1$ & $\mathrm{~S}$ & $\leq 1$ & $\mathrm{~S}$ & $\leq 1$ & $\mathrm{~S}$ \\
Cefepima & - & - & - & - & - & - & - & - & - & - & - & - & - & - \\
Imipenem & $\leq 0.25$ & $\mathrm{~S}$ & $\leq 0.25$ & $\mathrm{~S}$ & $\leq 0.25$ & $\mathrm{~S}$ & $\leq 0.25$ & $\mathrm{~S}$ & $\leq 0.25$ & $\mathrm{~S}$ & $\leq 0.25$ & $\mathrm{~S}$ & $\leq 0.25$ & $\mathrm{~S}$ \\
Meroperem & $\leq 0.25$ & $\mathrm{~S}$ & $\leq 0.25$ & $\mathrm{~S}$ & $\leq 0.25$ & $\mathrm{~S}$ & $\leq 0.25$ & $\mathrm{~S}$ & $\leq 0.25$ & $\mathrm{~S}$ & $\leq 0.25$ & $\mathrm{~S}$ & $\leq 0.25$ & $\mathrm{~S}$ \\
Amicacina & $\leq 2$ & $\mathrm{~S}$ & $\leq 2$ & $\mathrm{~S}$ & $\leq 2$ & $\mathrm{~S}$ & $\leq 2$ & $\mathrm{~S}$ & $\leq 2$ & $\mathrm{~S}$ & $\leq 2$ & $\mathrm{~S}$ & $\leq 2$ & $\mathrm{~S}$ \\
Gentamicina & $\leq 1$ & $\mathrm{~S}$ & $\leq 1$ & $\mathrm{~S}$ & $\leq 1$ & $\mathrm{~S}$ & $\leq 1$ & $\mathrm{~S}$ & $\leq 1$ & $\mathrm{~S}$ & $\leq 1$ & $\mathrm{~S}$ & $\leq 1$ & $\mathrm{~S}$ \\
Ácido nalidíxico & $\leq 4$ & $\mathrm{~S}$ & $\leq 2$ & $\mathrm{~S}$ & $\leq 2$ & $\mathrm{~S}$ & $\leq 2$ & $\mathrm{~S}$ & $\leq 2$ & $\mathrm{~S}$ & $\leq 2$ & $\mathrm{~S}$ & $\leq 2$ & $\mathrm{~S}$ \\
Ciprofloxacino & $\leq .25$ & $\mathrm{~S}$ & $\leq 0.25$ & $\mathrm{~S}$ & $\leq 0.25$ & $\mathrm{~S}$ & $\leq 0.25$ & $\mathrm{~S}$ & $\leq 0.25$ & $\mathrm{~S}$ & $\leq 0.25$ & $\mathrm{~S}$ & $\leq 0.25$ & $\mathrm{~S}$ \\
Nitrofurantoína & $\leq 16$ & $\mathrm{~S}$ & $\leq 16$ & $\mathrm{~S}$ & $\leq 16$ & $\mathrm{~S}$ & $\leq 16$ & $\mathrm{~S}$ & $\leq 16$ & $\mathrm{~S}$ & $\leq 16$ & $\mathrm{~S}$ & $\leq 16$ & $\mathrm{~S}$ \\
Trimetoprima/Sulfametoxazol & $\leq 20$ & $\mathrm{~S}$ & $\leq 20$ & $\mathrm{~S}$ & $\leq 20$ & $\mathrm{~S}$ & $\leq 20$ & $\mathrm{~S}$ & $\leq 20$ & $\mathrm{~S}$ & $\leq 20$ & $\mathrm{~S}$ & $\leq 20$ & $\mathrm{~S}$ \\
\hline & & & & & & & & & & & &
\end{tabular}



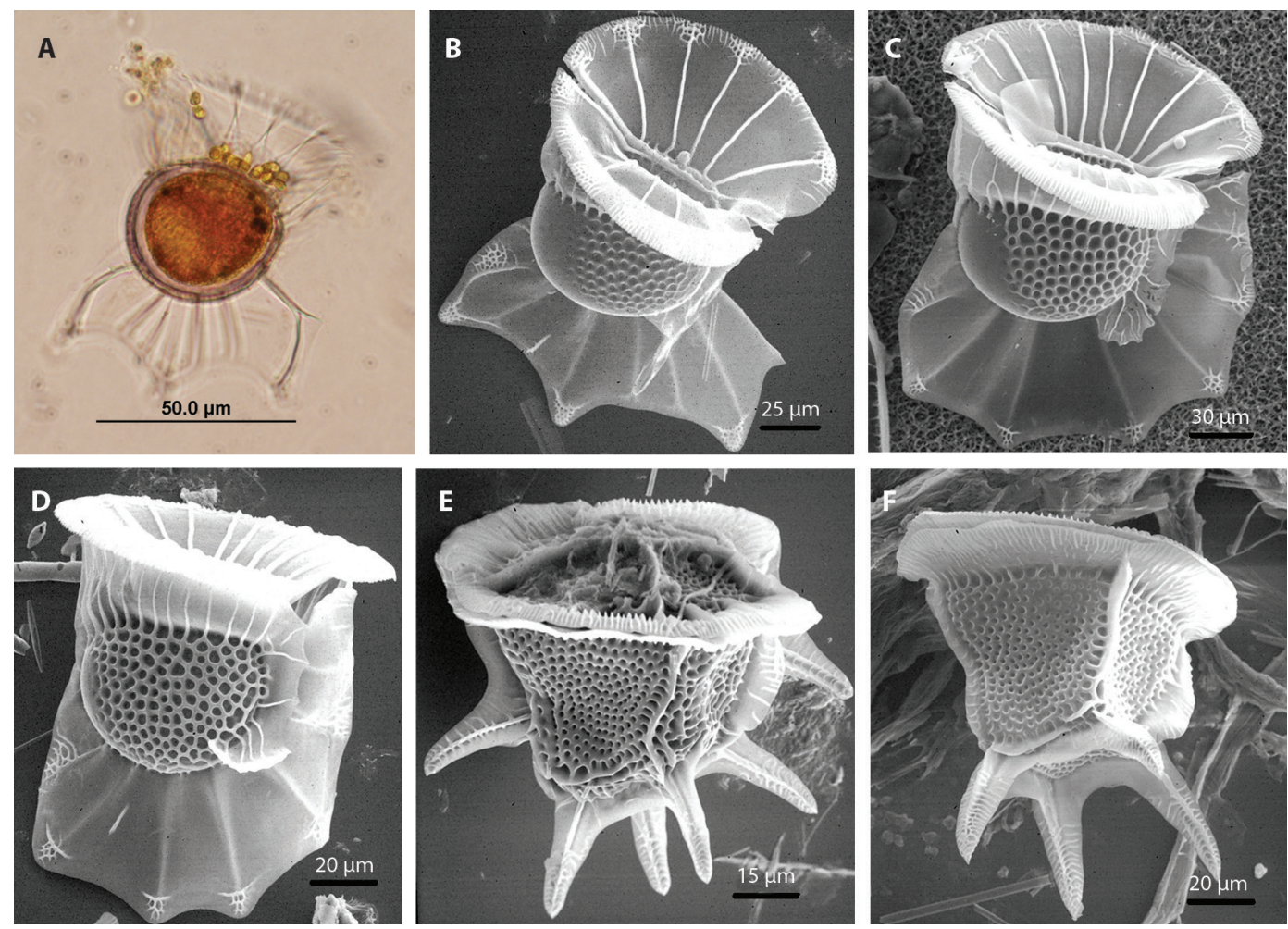

Fig. 3. Especies de dinoflagelados marinos luminiscentes observados en las muestras colectadas alrededor de la Isla del Coco. (A-B) Ornithocercus magnificus, (C-D) Ornithocercus steinii y (E-F) Ceratocorys horrida tomados con el microscopio electrónico de barrido.

Fig. 3. Species of bioluminescent marine dinoflagellates observed in samples collected around Cocos Island (A-B) Ornithocercus magnificus, (C-D) Ornithocercus steinii and (E-F) Ceratocorys horrida pictures by scanning electron microscopy.

El género Ornithocercus fue el más abundante en las muestras observadas.

\section{DISCUSIÓN}

Estudios previos referentes al reporte, aislamiento y caracterización de bacterias luminiscentes en aguas superficiales marinas de los géneros Vibrio y Photobacterium en la costa del Pacífico (Golfo de Nicoya) costarricense, son los presentados por Rojas-Alfaro, (2016) y Marín-Vindas et al. (2017). Sin embargo, nuestros hallazgos muestran por primera vez el aislamiento e identificación bioquímica de 7 aislados bacterianos luminiscentes de agua marina de la Isla del Coco
La caracterización bioquímica de los aislados luminiscentes mediante Vitek 2 mostró un alto nivel de confianza permitiendo la identificación bacteriana de las muestras ambientales tal y como lo confirman estudios realizados en cultivos, ecosistemas dulceacuícolas, marinos y muestras ambientales de reptiles silvestres (Romeu et al., 2010; Cardak, Özbek, \& Kebapçioğlu, 2015; Artavia-León et al., 2017). La identificación bioquímica indica que los aislados luminiscentes de este estudio se clasifican en el género Vibrio sp., reportado dentro del grupo de las bacterias luminiscentes (Bagordo, et al., 2012; Yaser et al., 2014; Gómez et al., 2017).

Las aislados de $V$. alginolyticus y $V$. parahaemolyticus, no pertenecen al clado Harveyi, 
TABLA 4

Observaciones de la actividad hemolítica realizadas en aislados luminiscentes aisladas de la Isla del Coco, Costa Rica

TABLE 4

Observations of hemolytic activity performed on luminescent strains isolated from Cocos Island, Costa Rica

\section{Código de cepa} Actividad hemolítica (mm)

\section{1}

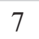

CL 2

CL 3

CL 4

CL 5

CL 6

CL 7

10

$7 *$

10

9

10

16

* Hemólisis parcial observada cuando la zona de crecimiento aparece rodeada de un halo de color verdoso mientras que la total es un halo totalmente transparente (Martins, 2010).

el cual es un complejo de bacterias que hasta el momento comprende 12 especies (V. rotiferianus, $V$. azureus, $V$. sagamiensis, $V$. jasicida, $V$. natriegens, $V$. mytili, $V$. harveyi, $V$. campbellii, $V$. owensii, $V$. rotiferianus, $V$. jasicida y $V$. hyugaensis). La complejidad de este grupo se debe a que los fenotipos y genotipos de esas especies son muy similares entre sí, por lo que su identificación por métodos bioquímicos y moleculares es compleja (Ke et al., 2018). Lo anterior evidencia que es necesaria una caracterización bioquímica y molecular de los aislamientos obtenidos en este estudio para confirmar la identidad de los aislados luminiscentes recuperados de la Isla del Coco.

La resistencia de las bacterias es un fenómeno natural en función de la evolución, adaptación y selección de las especies (Elmahdi, DaSilva, \& Parveen, 2016). La introducción de fármacos en ecosistemas acuáticos ha provocado un gran efecto secundario en el ambiente, la propagación de genes de resistencia a antibióticos (Song et al., 2016). El alto porcentaje de resistencia a la ampicilina (57\%) es consistente como lo reportado por (Elmahdi et al., 2016) donde indica que $V$. alginolyticus y $V$. parahaemolyticus mostraron resistencia a este antibiótico, además la ampicilina forma parte de la lista de antibióticos que las bacterias del género Vibrio de origen marino muestran resistencia (Lee et al., 2018).

Una parte importante de la dispersión y evolución de las bacterias resistentes a los antimicrobianos depende del agua. En ecosistemas acuáticos las bacterias de diferentes orígenes (animal, humana y ambiental) son capaces de mezclarse y desarrollar mecanismos de resistencia como consecuencia de intercambios promiscuos de elementos genéticos móviles o de la recombinación de genes Asimismo, los antimicrobianos y metales pesados son liberados en el agua y sus residuos pueden ejercer presiones selectivas en comunidades acuáticas, resultando en resistencia a los agentes antimicrobianos (Torres et al., 2016). La resistencia no solo se adquiere por exposición al compuesto, sino también a través de mecanismos de defensa propios de la bacteria, como la bomba de flujo que expulsa la sustancia extraña, o bien la resistencia adquirida por medio de plásmidos y de betalactamasas de amplio espectro (enzimas bacterianas que hidrolizan y generan resistencia a los antibióticos de tipo $\beta$-lactámicos) (Lee et al., 2018).

El alto porcentaje de sensibilidad para los 12 antibióticos con la excepción de las aislados CL 3, CL 4, CL 5 y CL 6 que fueron resistentes a la ampicilina (Tabla 3 ), coincide con lo reportado por Elmahdi et al. (2016) y por Lee et al. (2018), quienes afirman que $V$. alginolyticus y V. parahaemolyticus y otras bacterias del género Vibrio aislados del medio marino evidenciaron sensibilidad ante dichos antibióticos.

La susceptibilidad de las demás aislados puede atribuirse a que la ampicilina es un antibiótico de tipo $\beta$-Lactámico de amplio espectro con un efecto bactericida lento, cuyo modo de acción es la inhibición de la transpeptidación, la última fase de la síntesis de la pared celular, causando un efecto letal en la bacteria (Dörr et al., 2016). Con respecto al resto de antibióticos ensayados, la alta sensibilidad es compatible con la baja exposición a drogas de uso restringido al ambiente hospitalario, de 
manera consistente con otros estudios similares (Torres et al., 2016). La determinación de la actividad hemolítica de bacterias luminiscentes del género Vibrio obtenida en este estudio (Tabla 4) concuerda con lo reportado (Cai \& Zhang, 2018), indicando que uno de los efectos de la infección causada por estos microorganismos es la hemólisis en animales marinos como peces, camarones e incluso en bivalvos (Silva et al., 2018).

Las aguas alrededor de la Isla del Coco presentan el fenómeno de la bioluminiscencia, que puede ser observado por las noches, en gran parte debido a la presencia de los géneros de dinoflagelados Ornithocercus y Ceratocorys, que siempre estuvieron presentes en las muestras. Muchas de las especies observadas nunca se han reportado en aguas costeras de Costa Rica, ya que esta isla es meramente oceánica y recibe la influencia de las aguas provenientes del hemisferio sur, por lo que la mayoría de los organismos encontrados son meramente oceánicos (Vargas-Montero, Morales, \& Cortés, 2012). La presencia de especies de dinoflagelados luminiscentes en las aguas alrededor de la Isla del Coco es un fenómeno que se observa en todas las épocas del año, y las especies pueden variar dependiendo de la disponibilidad de nutrimentos y de las condiciones oceanográficas, lo que refleja su estado o actividad metabólica (Sweeney, 1987). Este fenómeno se mantiene tanto con la estimulación química de los microorganismos como al estimular mecánicamente el agua, lo que aumenta aún más su visibilidad a diferentes profundidades. Estudios recientes, han demostrado que la bioluminiscencia es un factor muy importante en la determinación de la presencia de dinoflagelados tóxicos en el agua y que su actividad además de ser proporcional al número de células puede variar de acuerdo con las condiciones fisiológicas del organismo (Uribe \& Montecino, 2007).

Estudios de monitoreo de luminiscencia acuática tendrían una afectación social-cultural de forma directa con los pobladores de las zonas costeras del país, ya que actualmente existen varias agencias turísticas que operan visitas a sitios de bioluminiscencia reportadas en el todo el Pacífico de Costa Rica, siendo esta una alternativa de turismo rural comunitario en miras de fortalecimiento en cuanto a capacitación y educación ambiental. En conclusión, este trabajo expone el primer registro de aislamiento y caracterización bioquímica automatizada de bacterias luminiscentes de agua marina superficial (profundidad: $1 \mathrm{~m}$ ) y mediante buceo (profundidad: $20 \mathrm{~m}$ ) obtenidas de la Isla del Coco, Costa Rica. Además de la relevancia en la ampliación de las zonas de estudio, aislamiento, caracterización bioquímica y molecular con el fin de explorar la diversidad que presentan los microorganismos luminiscentes en ambientes acuáticos de Costa Rica. La gran biodiversidad de especies de fitoplancton marino que se presentan en la isla, aunada a la abundancia de dinoflagelados sobre los parches de coral en las diferentes bahías, evidencian además de la utilización de mecanismos de defensa, que su población es abundante y está en crecimiento exponencial, generando un espectáculo visual que podría utilizarse para determinar cuáles especies potencialmente productoras de floraciones algales se encuentran presentes en el agua.

Declaración de ética: los autores declaran que todos están de acuerdo con esta publicación y que han hecho aportes que justifican su autoría; que no hay conflicto de interés de ningún tipo; y que han cumplido con todos los requisitos y procedimientos éticos y legales pertinentes. Todas las fuentes de financiamiento se detallan plena y claramente en la sección de agradecimientos. El respectivo documento legal firmado se encuentra en los archivos de la revista.

\section{AGRADECIMIENTOS}

Externamos agradecimientos a Jorge Cortés Núñez, investigador del Centro de Investigación en Ciencias del Mar y Limnología de la Universidad de Costa Rica y a Luis Acosta Vargas, académico del Instituto Tecnológico de Costa Rica por colaborar en la realización 
de la visita al PNIC y al procesamiento de las muestras. A David Morúa Perez por su colaboración durante la gira de campo. A Yeimy Ramírez y Jhonatan Campos del Laboratorio de Bacteriología Médica de la Facultad de Microbiología de la Universidad de Costa Rica, por su apoyo técnico en la caracterización bioquímica y fenotípica de las aislados bacterianas. A Sergio Cambronero Solano por la elaboración del mapa. A Alberto Muñoz del barco Okeanos Aggressor II por la colecta de las muestras de buceo. A Guillermo Blanco Segura por las capturas fotográficas, y especialmente a todos los administradores, guardaparques del PNIC por toda la ayuda y disposición antes, durante y después de la visita.

\section{RESUMEN}

Introducción: La bioluminiscencia es la capacidad de ciertos organismos para transformar la energía química en energía lumínica mediante varios procesos bioquímicos. Objetivo: el aislamiento e identificación por primera vez de bacterias luminiscentes en agua marina superficial y la identificación de dinoflagelados luminiscentes marinos del Parque Nacional Isla del Coco, Costa Rica. Metodología: Se colectaron muestras de agua marina obtenida por buceo a $20 \mathrm{~m}$ y a nivel superficial de 13 sitios en la Isla del Coco, Costa Rica. Por otra parte, se analizaron muestras de fitoplancton colectadas desde la superficie hasta los 30 $\mathrm{m}$ de profundudad en los alrededores de 8 sitios de la Isla del Coco, y se determinaron varias especies luminiscentes pertenecientes a los géneros Ornithocercus y Ceratocorys. Resultados: Se logró obtener 7 aislados bacterianos luminiscentes, se identificaron y caracterizaron bioquímicamente mediante una plataforma automatizada (Vitek) con altos niveles de confianza, se ubicaron taxonómicamente dentro del género Vibrio, 2 especies: $V$. alginolyticus y $V$. parahaemolyticus, además, algunos aislados presentaron resistencia al antibiótico ampicilina y 100\% capacidad hemolítica. Esta investigación muestra evidencia de la presencia de especies microscópicas marinas en Isla del Coco, Costa Rica, capaces de presentar el fenómeno de la luminiscencia, por lo que profundizar en su estudio sería relevante en cuanto a la importancia de estos microorganismos en la producción de metabolitos secundarios y como indicadores de floraciones algales nocivas, por lo que se hace necesario realizar más investigación científica para determinar su potencial biotecnológico. Conclusiones: De la misma forma, los resultados obtenidos en esta investigación sugieren expandir las localidades de colecta y aislamientos de microorganismos luminiscentes, acompañado de una caracterización bioquímica y molecular, con el fin de explorar la diversidad microbiana asociada a eventos de luminiscencia y determinar los ambientes en el que estas especies se desarrollan.

Palabras clave: Luminiscencia, bacterias marinas, dinoflagelados, bacilos Gram-negativos, Isla del Coco.

\section{REFERENCIAS}

Alvarado, J., Herrera, B., Corrales, L., Asch, J., \& Paaby, P. (2011). Identificación de las prioridades de conservación de la biodiversidad marina y costera en Costa Rica. Revista de Biología Tropical, 59(2), 829-842.

Artavia-León, A., Romero-Guerrero, A., Sancho-Blanco, C., Rojas, N., \& Umaña-Castro, R. (2017). Diversity of aerobic bacteria isolated from oral and cloacal cavities from free-living snakes species in Costa Rica rainforest. International Scholarly Research Notices, $2017,8934285$.

Bagordo, F., Serio, F., Lugoli, F., Grassi, T., Idolo, A., Gabutti, G., \& De Donno, A. (2012). Phenotypic characterization of culturable marine luminous bacteria isolated from coastal waters of the southern Adriatic Sea (Otranto, Italy). Ciencias Marinas, 38(4), 599-608.

Cai, Q., \& Zhang, Y. (2018). Structure, function and regulation of the thermostable direct hemolysin (TDH) in pandemic Vibrio parahaemolyticus. Microbial Pathogenesis, 123, 242-245.

Cardak, M., Özbek, Ö., \& Kebapçioğlu, T. (2015). Seasonal abundance and diversity of culturable heterotrophic bacteria in relation to environmental factors in the Gulf of Antalya, Eastern Mediterranean, Turkey. World Journal of Microbiology and Biotechnology, 31(4), 569-582.

Chunga, J. (2018). Vibrio cholerae y Vibrio parahaemolyticus toxigénicos en cuerpos de agua de Piura. (Tesis de doctorado). Universidad Nacional de Piura, Perú.

Cortés, J. (2012). Marine biodiversity of an Eastern Tropical Pacific oceanic island, Isla del Coco, Costa Rica. Revista de Biología Tropical, 60(Supplement 3), 131-185.

Cortés, J. (2017). Marine biodiversity baseline for Área de Conservación Guanacaste, Costa Rica: published records. ZooKeys, 652, 129-179.

Cortés, J. (2016a). Isla del Coco: Coastal and marine ecosystems. In M. Kappelle (Ed.), Costa Rican Ecosystems (pp. 162-191). Chicago and London: University of Chicago Press.

Cortés, J. (2016b). The Caribbean coastal and marine ecosystems. In M. Kappelle (Ed.), Costa Rican Ecosystems (pp. 591-617). Chicago and London: University of Chicago Press. 
Cortés-Núñez, J., Acuña-González, J, Rodríguez-Lizano, O. G., Morales-Ramírez, A., \& Alfaro-Martínez, E. J. (2009). Informe final del Proyecto No. 808-A7-520, Conocimiento y gestión de medios marinos y coralinos del Área de Conservación Marino Isla del Coco. Recuperado de http://hdl.handle.net/10669/436

Dörr, T., Alvarez, L., Delgado, F., Davis, M., Cava, F., \& Waldor, M. (2016). A cell wall damage response mediated by a sensor kinase/response regulator pair enables beta-lactam tolerance. Proceedings of the National Academy of Sciences, 113(2), 404-409.

Elmahdi, S., DaSilva, L. V., \& Parveen, S. (2016). Antibiotic resistance of Vibrio parahaemolyticus and Vibrio vulnificus in various countries: a review. Food Microbiology, 57, 128-134.

Gómez, D., Castro, R., González, S., Rodríguez, M., Guilarte, E., Valcárcel, C., \& Gallardo, G. (2017). Phenotypic characterization and molecular identification of a luminescent marine bacterum isolated from the new shelf of Cuba. Biotecnia, 19(3), 3-10.

Kahlke,T., \& Umbers, K. (2016). Bioluminescence. Current Biology, 26, 313-314.

Khambholja, D. B., \& Kalia, K. (2016). Isolation and characterization of arsenic tolerent marine bacteria from South Gujarat coast Near Vapi, India. Journal of Cell and Tissue Research, 16(1), 5359.

Ke, H., Liu, D., Ogura, Y., Hayashi, T., Urbanczyk, H., \& Tsai, I. (2018). Tracing genomic divergence of Vibrio bacteria in the Harveyi clade. Journal of Bacteriology, 200(15), e00001-18.

Kola, S. G., \& Masilamani, S. (2017). Isolation and characterization of bioluminescent bacteria from marine organisms. Indian Journal of Geo Marine Sciencies, 46(4), 797-801.

Lee, L., Ab, N., Law, J, Wong, S., \& Letchumanan, V. (2018). Discovery on antibiotic resistance patterns of Vibrio parahaemolyticus in Selangor reveals carbapenemase producing Vibrio parahaemolyticus in marine and freshwater fish. Frontiers in Microbio$\log y, 9,2513$.

Marín-Vindas, C., Vega-Corrales, L., \& Rojas-Alfaro, R. (2015, Julio). Distribución y abundancia de bacterias luminiscentes y su potencial como indicadoras de contaminación en el Golfo de Nicoya, Costa Rica. En M. Somohano (Presidencia), VI Jornada de Investigación sobre el Pacífico Costarricense. Universidad de Costa Rica, Sede Regional del Pacífico, Puntarenas, Costa Rica.

Marín-Vindas, C., Vega-Corrales, L., Umaña-Castro, R., \& Rojas-Alfaro, R. (2015, Noviembre). Bacterias luminiscentes y su potencial como indicadoras de contaminación en el Golfo de Nicoya, Costa Rica. En G. García (Presidencia), Simposio III Conservación y Biodiversidad, Simposio llevado a cabo en el X
Congreso de Ciencias del Mar, MarCuba, Habana, Cuba.

Marín-Vindas, C., Vega-Corrales, L., Umaña-Castro, R., \& Rojas-Alfaro, R. (2017, Septiembre). Characterization of culturable marine luminescent bacteria isolated from the Gulf of Nicoya, Costa Rica. En I. Berman (Presidencia), 15th Symposium on Aquatic Microbial Ecology, SAME15, Croatia.

Martins, P. (2010). Prospecção de enzimas de interesse biotecnológico de Vibrios associados a corais endêmicos brasileiros. (Tesis de doctorado). Universidad de Católica de Brasília, Brasil.

Mazia, D., Schatten, G., \& Sale, W. (1975). Poly-lysine method: Adhesion of cells to surfaces coated with polylysine. Application to electron microscopy. Journal of Cell Biology, 66, 198-200.

Rojas-Alfaro, R. (2016). Potencial biotecnológico de bacterias luminiscentes aisladas del Golfo de Nicoya, Costa Rica. (Tesis de Licenciatura), Universidad Nacional, Heredia, Costa Rica.

Romeu, B., Salazar, P., Navarro, A., Lugo, D., Hernández, U., Rojas, N., \& Eslava, C. (2010). Utilidad del sistema VITEK en la identificación y determinación de la susceptibilidad antimicrobiana de bacterias aisladas de ecosistemas dulceacuícolas. Revista CENIC Ciencias Biológicas, 41, 1-9.

Rusch, D., Halpern, A., Sutton, G., Heidelberg, K., Williamson, S., Yooseph, S., ... \& Beeson, K. (2007). The Sorcerer II global ocean sampling expedition: northwest Atlantic through eastern tropical Pacific. Public Library of Science Biology, 5(3), 395-431.

Silva, I., de Souza, C., Saraiva, M., de Oliveira, T., de Sousa, O., \& Evangelista-Barreto, N. (2018). Antimicrobial resistance and potential virulence of Vibrio parahaemolyticus isolated from water and bivalve mollusks from Bahia, Brazil. Marine Pollution Bulletin, 131, 757-762.

Song, C., Zhang, C., Fan, L., Qiu, L., Wu, W., Meng, S., ... \& Chen, J. (2016). Occurrence of antibiotics and their impacts to primary productivity in fishponds around Tai Lake, China. Chemosphere, 161, 127-135.

Sweeney, B. M. (1987). Bioluminiscence and circadian rythms. In F. J. R. Taylor (Ed.), The Biology of Dinoflagellates (pp. 269-281). Oxford, UK: Blackwell Scientific Publications.

Uribe, P., \& Montecino, V. (2007). Estudios preliminares de la bioluminiscencia como herramienta para la detección temprana de dinoflagelados tóxicos en los canales y fiordos de la región XI. Ciencia y Tecnología del Mar, 30(2).

Vargas-Montero, M., \& Freer, E. (2003). Co-ocurrence of diferent morphotypes of Pyrodinium bahamense during an extensive bloom in the Gulf of Nicoya, Costa Rica. In A. Villalba, B. Reguera, J. Romalde, \& R. Beiras (Eds.), Proccedings of the 4th International 
Conference on Molluscan Shellfish Safety (pp. 211217). Santiago de Compostela, Spain.

Vargas-Montero, M., Freer, E., Guzmán, J. C., \& Vargas, J. (2008). Harmful blooms by noxious dinoflagellates in the Pacific coast of Costa Rica. Hidrobiológica, 18(1): $15-23$

Vargas-Montero, M., Morales, A., \& Cortés, J. (2012). Primer informe del género Gambierdiscus (Dinophyceae) y otros dinoflagelados bentónicos en el Parque Nacional Isla del Coco, Costa Rica, Pacífico Tropical Oriental. Revista de Biología Tropical, 60(Supplement 3), 187-199.

Vega-Corrales, L., Marín-Vindas, C., \& Rojas-Alfaro, R. (2014, Julio). Bacterias luminiscentes como alternativa para la evaluación de la contaminación marino costera en Costa Rica. En R. Ramírez (Presidencia), VIII Congreso Nacional de Biología, San José, Costa Rica.

Villalobos-Solé, C. (1967). Bioluminiscencia. O'Bios Costa Rica, 2(1), 16-17.

Víquez, R., \& Hargraves, P. (1995). Annual cycle of potentially harmful dinoflagellates in the Golfo de Nicoya, Costa Rica. Bulletin of Marine Science, $57(2), 467-475$.

Yaser, N., Foong, M., Mohd, A., \& Izni, I. (2014). Isolation and identificaction of bioluminescent bacteria in squid and water of Malaysia. Journal of Advances in Agricultural and Environment, 1(2), 225-228.

Watson, L., Mckee, A., \& Merrell, B. (1980). Preparation of Microbiological Specimens for Scanning Electron Microscopy. Scanning Electron Microscopy/1980/II. SEM Inc., AMF O`Hare, Chicago.

Wehrtmann, I., Cortés, J., \& Echeverría-Sáenz, S. (2009). Marine biodiversity of Costa Rica: perspectives and conclusions. Chapter V. In I. S. Wehrtmann, \& J. Cortés (Eds.), Marine Biodiversity of Costa Rica, Central America (pp. 521-533). Monographiae Biologicae 86. Berlin: Springer \& Business Media B. V.

Wehrtmann, I., \& J. Cortés (Eds.). (2009). Marine Biodiversity of Costa Rica, Central America. Monographiae Biologicae 86. Berlin: Springer \& Business Media B. V.

Witze, A. (2018). Hidden lives of deep-sea animals. Nature, 561, 296-297. 